\title{
Image multiplexing using residue number system coding over MIMO-OFDM communication system
}

\author{
M. I. Youssef, A. E. Emam, M. Abd Elghany
}

Electrical Engineering Department, Faculity of Engineering, Al- Azhar University, Egypt

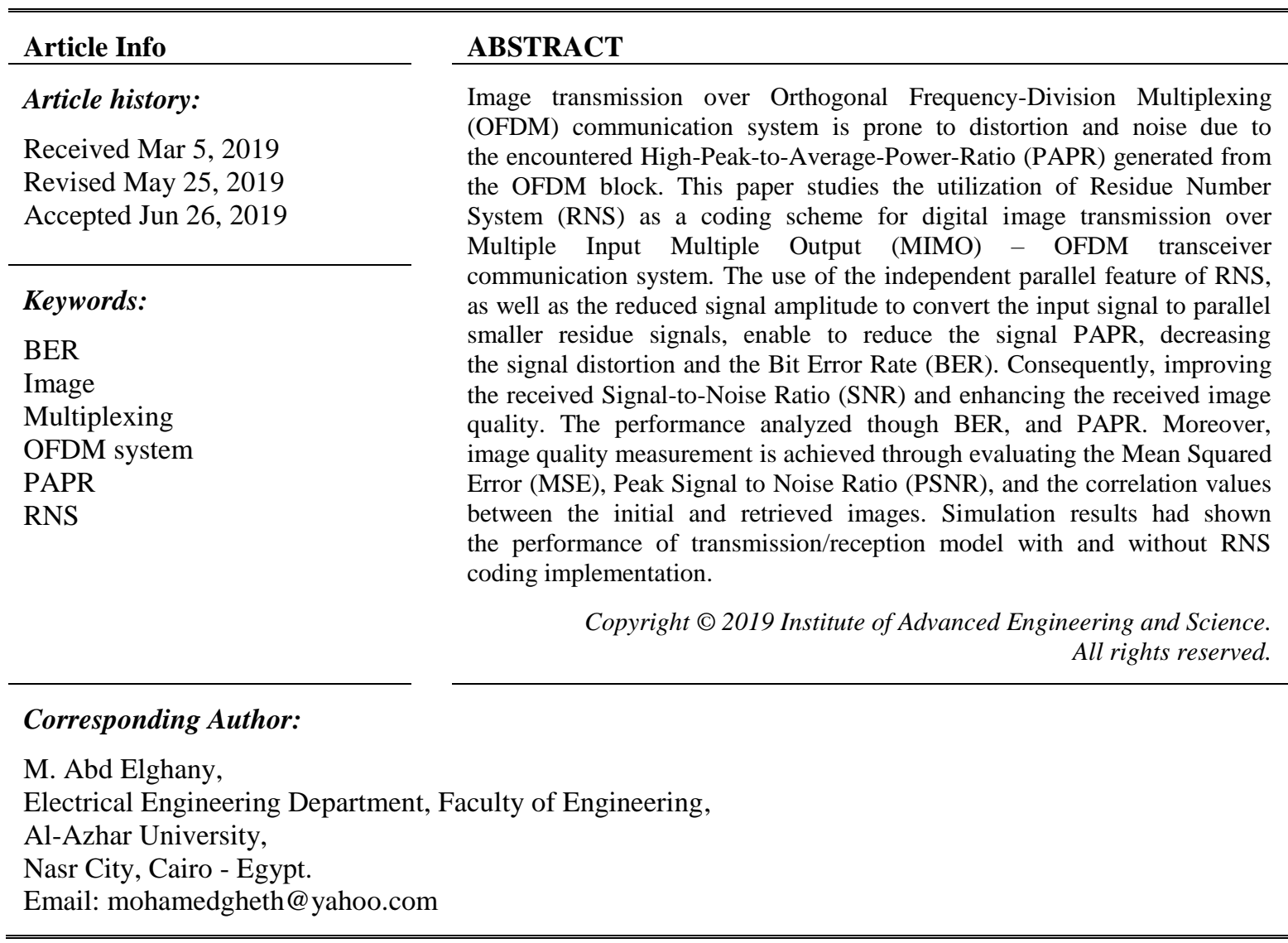

\section{INTRODUCTION}

A MIMO-OFDM system is a high data-rate communication system capable of providing a high quality of service along with efficient usage of frequency resources over various fading conditions [1-3]. The basic principle for this system is the utilization of multiple numbers of sub-carriers to decrease the data rates which has to carry, and then data is transmitted over multiple antenna system to achieve diversity gain.

Still, the communication system performance is severally degraded due to the high generated signal PAPR that when passed through the system power amplifiers produce signal distortion and noise due to the amplification in the non-linear device range. These drawbacks would induce significant signal distortions that increase the difficulty at the receiver side to detect correctly the received transmission, and add up in the design complexity of the RF amplifiers.

Previous literature $[4,5]$ provided several power reduction techniques which are categorized based on whether the transmitted signal is distorted or not as: Clipping/filtering approach and coding approach. In this paper the RNS is used as a coding technique to decrease the generated PAPR signal in OFDM systems and enhance the quality of received image, taking benefit from the residue number properties as independent parallel communication, lower signal amplitude and high power spectral density, Moreover, the RNS is used for transmission error detection and correction through adding additional moduli as a redundant symbol and this is referred to as a Redundant Residue Number System (RRNS). 
In section 2, literature review for RNS and RRNS techniques are presented, which is followed in section 3 and 4 by providing the analytical and theoretical explanation on the approach the RNS is used to reduce the PAPR, and to detect and correct transmission errors respectively. Section 5, present the MIMOOFDM communication system block sets including the RNS coding stage, and in section 6, a description on the key elements used to evaluate the reception quality of the received image are stated. In Section 7 , the simulation results are presented to measure the system performance, and finally, in section 8 , the conclusion has been provided.

\section{LITERATURE REVIEW}

In this section a review for both the Residue Number System (RNS) and the Redundant Residue Number System (RRNS) are being provided.

\subsection{Residue number system}

RNS are a set of integer numbers with carry-free, parallel, and fast-processing properties that is used in various mathematical operations [6]. Through this system an RNS sequence $\left\{r_{i}\right\}$ is the reminder of dividing the input message ' $\mathrm{N}$ ' by the selected set of ' $v$ ' relative primes moduli $\left\{\mathrm{m}_{\mathrm{i}}\right\}$, which is shown in (1);

$$
\mathrm{r}_{\mathrm{i}}=\mathrm{N}\left(\bmod \mathrm{m}_{\mathrm{i}}\right)
$$

where, the symbols dynamic range is shown in (2);

$$
\mathrm{M}_{\mathrm{I}}=\prod \mathrm{m}_{\mathrm{i}}
$$

At receiver side, the Mixed Radix Conversion (MRC) technique [7] is used to recover symbols. So, for a set of pair-wise relatively prime moduli $\left\{\mathrm{m}_{1}, \mathrm{~m}_{2}, \ldots, \mathrm{m}_{\mathrm{n}}\right\}$ and a residue state $\left\{\mathrm{r}_{1}, \mathrm{r}_{2}, \ldots . \mathrm{r}_{\mathrm{n}}\right\}$ of a number $\mathrm{X}$, that number can be uniquely represented in mixed-radix form as shown in next:

$$
X=\left\{z_{1}, z_{2}, \ldots, z_{n}\right\}
$$

and;

$$
\mathrm{X}=\mathrm{z}_{1}+\mathrm{z}_{2} \mathrm{~m}_{1}+\mathrm{z}_{3} \mathrm{~m}_{2} \mathrm{~m}_{1}+\ldots . .+\mathrm{z}_{\mathrm{n}} \mathrm{m}_{\mathrm{n}-1} \mathrm{~m}_{\mathrm{n}-2} \ldots . \mathrm{m}_{1} ; 0 \leq \mathrm{z}_{\mathrm{i}} \leq \mathrm{r}_{\mathrm{i}}
$$

where; $z_{\mathrm{i}}$ is represented as function of the moduli and residue representations as shown in Table 1 .

Table 1. Representation of $z_{i}$

\begin{tabular}{cl}
\hline Parameter & \multicolumn{1}{c}{ Representation } \\
\hline $\mathrm{z}_{1}$ & $=\mathrm{r}_{1}$ \\
$\mathrm{z}_{2}$ & $=\|\left.\left.\mathrm{m}_{1}{ }^{-1}\right|_{\mathrm{m} 2}\left(\mathrm{r}_{2}-\mathrm{z}_{\mathrm{Z}}\right)\right|_{\mathrm{m} 2}$ \\
$\mathrm{z}_{3}$ & $=\|\left.\left(\mathrm{m}_{2} \mathrm{~m}_{1}\right)^{-1}\right|_{\mathrm{m} 3}\left(\mathrm{r}_{3}-\left.\left(\mathrm{z}_{2} \mathrm{~m}_{1}+\mathrm{z}_{1}\right)\right|_{\mathrm{m} 3}\right.$ \\
$\mathrm{z}_{\mathrm{n}}$ & $=\|\left.\left.\left(\mathrm{m}_{\mathrm{n}} \ldots \ldots . \mathrm{m}_{2} \mathrm{~m}_{1}\right)^{-1}\right|_{\mathrm{mn}}\left(\mathrm{r}_{\mathrm{n}}-\mathrm{z}_{\mathrm{n}-1} \mathrm{~m}_{\mathrm{n}-2} \ldots \ldots \mathrm{z}_{2} \mathrm{~m}_{1}+\mathrm{z}_{1}\right)\right|_{\mathrm{mn}}$ \\
\hline
\end{tabular}

\subsection{Redundant residue number system}

For the detection and correction of transmission errors, additional RNS moduli are utilized as a redundant symbol with the RNS moduli set. The new moduli set are called Redundant Residue Number System (RRNS). Where, through this scheme, each redundant moduli selected to be greater than any of the other chosen moduli set and don't contribute in defining the system dynamic range.

So, an RRNS obtained by appending an additional $(\mathrm{u}-\mathrm{v})$ set of moduli $\mathrm{m}_{\mathrm{v}+1} ; \mathrm{m}_{\mathrm{v}+2} ; \ldots . . ; \mathrm{m}_{\mathrm{u}}$, such that $\mathrm{m}_{\mathrm{v}+\mathrm{j}} \geq \max \left\{\mathrm{m}_{1} ; \mathrm{m}_{2} ; \ldots \ldots ; \mathrm{m}_{\mathrm{v}}\right\}$, is referred to as a redundant modulus to the introduced RNS in section 2.1 , in order to form an RRNS of ' $u$ ' positive, pairwise relative prime moduli set $[8,9]$. For error correction using the MRC method, a combination of two redundant moduli are used to test every information moduli. Where, it is able to identify and correct the erroneous bit through the performed test [10]. 


\section{RNS AS PAPR REDUCTION TECHNIQUE}

Let the input data symbols on the subcarriers as $d_{i}(i=0,1, \ldots, M-1)$, where $M$ is the number of subcarriers. The output after inverse discrete Fourier transform is $s_{\mathrm{k}}$, as shown in (5);

$$
\mathrm{s}_{\mathrm{k}}=(1 / \mathrm{M}) \sum \mathrm{d}_{\mathrm{i}} \mathrm{e}^{\mathrm{j} 2 \pi \mathrm{i} / \mathrm{M}}, \text { for } 0 \leq \mathrm{k} \leq \mathrm{M}-1
$$

So, the PAPR of OFDM-based scheme for M sub-channel is provided in (6);

$$
\mathrm{PAPR}_{\mathrm{ofdm}}=\frac{\max \left\{|x(k)|^{2}\right\}}{\mathrm{E}\left\{|x(k)|^{2}\right\}}=\frac{\max \left\{\left|\sum_{i=0}^{M-1} d_{i} \exp \left(j \frac{2 \pi i k}{M}\right)\right|^{2}\right\}}{\mathrm{E}\left\{\left|\sum_{i=0}^{M-1} d_{i} \exp \left(j \frac{2 \pi i k}{M}\right)\right|^{2}\right\}}=\frac{\max \left\{\left|\sum_{i=0}^{M-1} d_{i} \exp \left(j \frac{2 \pi i k}{M}\right)\right|^{2}\right\}}{\frac{1}{\mathrm{M}}\left\{\left|\sum_{i=0}^{M-1} d_{i} \exp \left(j \frac{2 \pi i k}{M}\right)\right|^{2}\right\}}
$$

So, as seen from (6), the PAPR is proportional to the number of sub-carriers present in the IFFT stage, where data symbols across subcarriers can add up to produce a high peak value signal.

Now, inserting RNS pre-coding phase, it is seen that for residue $\left\{\mathrm{r}_{\mathrm{mv}, 0}, \mathrm{r}_{\mathrm{mv}, 1}, \ldots, \mathrm{r}_{\mathrm{mv},(\mathrm{N}-1)}\right\}$ transmitted over ' $v$ ' IFFT sub-channels corresponding to number of modulus set $m_{v}(v=1,2, \ldots V)$. Where, the output corresponding to the modulus $m_{v}$ residue sub-channel after IFFT is represented as seen in (7);

$$
\mathrm{s}_{\mathrm{k}, \mathrm{mv}}=\mathrm{s}(\mathrm{kT} / \mathrm{M})=\sum \mathrm{r}_{\mathrm{mv}} \mathrm{e}^{\mathrm{j} 2 \pi \mathrm{i} / \mathrm{M}}, \text { for } 0 \leq \mathrm{k} \leq \mathrm{M}-1, \text { for } 0 \leq \mathrm{i} \leq \mathrm{M}-1
$$

So, the PAPR of RNS-OFDM based scheme for ' $v$ ' sub-channel is provided in (8);

$$
\mathrm{PAPR}_{\text {rns-ofdm }}=\text { v. } \frac{\max \left\{\left|\sum_{\mathrm{i}=0}^{\mathrm{M}-1} \mathrm{r}_{\mathrm{mv}, 1} \exp \left(\mathrm{j} \frac{2 \pi \mathrm{ik}}{\mathrm{M}}\right)\right|^{2}\right\}}{\mathrm{E}\left\{\left|\sum_{\mathrm{i}=0}^{\mathrm{M}-1} \mathrm{r}_{\mathrm{mv}, 1} \exp \left(\mathrm{j} \frac{2 \pi \mathrm{ik}}{\mathrm{M}}\right)\right|^{2}\right\}} ;
$$

Comparing (6) and (8), it is seen that as RNS moduli set chosen smaller than the original signal and as the residue number is less than the moduli set, it is seen that the amplitude of RNS-OFDM signal ' $\mathrm{r}_{\mathrm{mv}}$ " is always less than that of the OFDM signal ' $d_{i}$ '. Also, based on the selection of the RNS moduli set ' $v$ ' it is designated to be less than number of subcarriers used in OFDM scheme ' $M$ '. Consequently, the generated PAPR in RNS-OFDM system is always less than that present in OFDM technique.

\section{RNS ERROR DETECTION AND CORRECTION ALGORITHM}

Through the implementation of RNS as a Forward Error Correction (FEC) scheme, this system is able to perform self-checking, error-detection and correction. This technique is evaluated with respect to conventional FEC techniques that do not require a handshaking process between Transmit/Receive systems, as Block Error correction codes, or Convolutional Error correction codes [11, 12].

In Figure 1, a flow chart indicating the procedure taken to detect and correct the transmission errors through the use of the redundant residue numbers is provided. Where, the system is able to detect and recover ' $\mathrm{N}$ ' erroneous integers by any ' $v$ ' out of ' $u$ ' residue digits using their related moduli. Furthermore, RRNS approach is the only one that is capable of using the same arithmetic module for generating both original information part and parity part of a RRNS code word [10, 13].

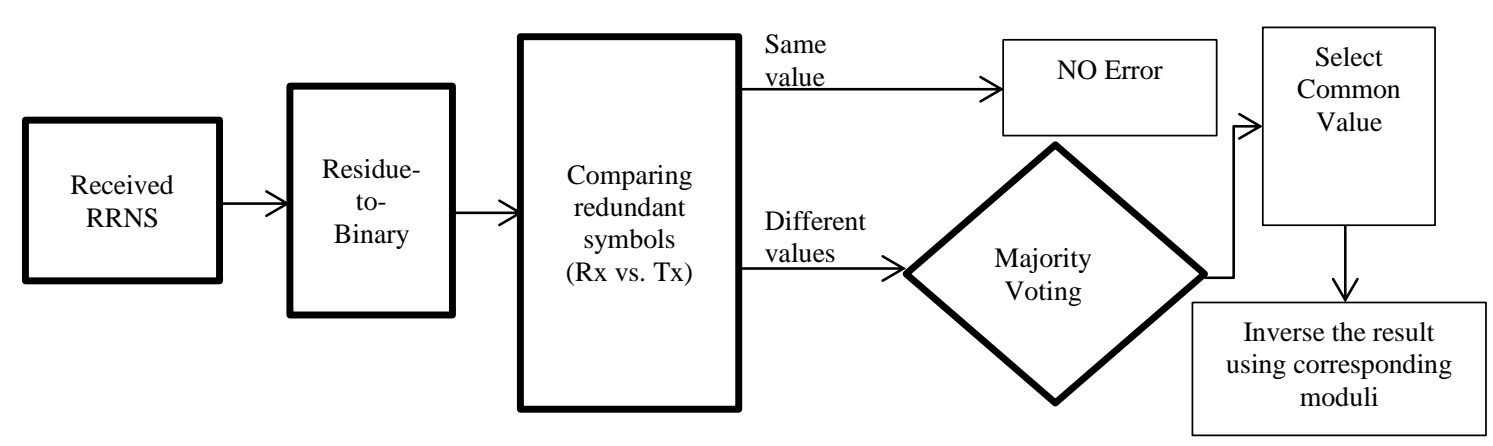

Figure 1. Error detection and correction algorithm 
In Figure 1 received symbols converted back to binary numbers and compared to the expected ones. Through this comparison the system detect occurrence of an error in the transmitted message. Then, for the correction stage, the modulus that generated the error must be identified either in $\mathrm{m}_{3}, \mathrm{or}_{2}$ or $\mathrm{m}_{1}$. Using the MRC method [6], a test on each information moduli with the two redundant moduli is performed to identify and correct the bit which generated the error [10,11]. Consequently, through this detection and correction algorithm, the error would be detected and corrected without the need to re-transmit again the information.

\section{SYSTEM MODEL}

The communication system, shown in Figure 2 is initialized with an image as an input source, which is transferred to binary data then converted to residue number system and protected from errors by adding parity residue symbols using the RRNS encoding algorithm. The packet is modulated, coded through the Space-Time Block Coding (STBC) encoder, passed to a Serial-To-Parallel (S/P) converter for parallel transmission, and then passed through an IFFT block then to the transmission antenna.

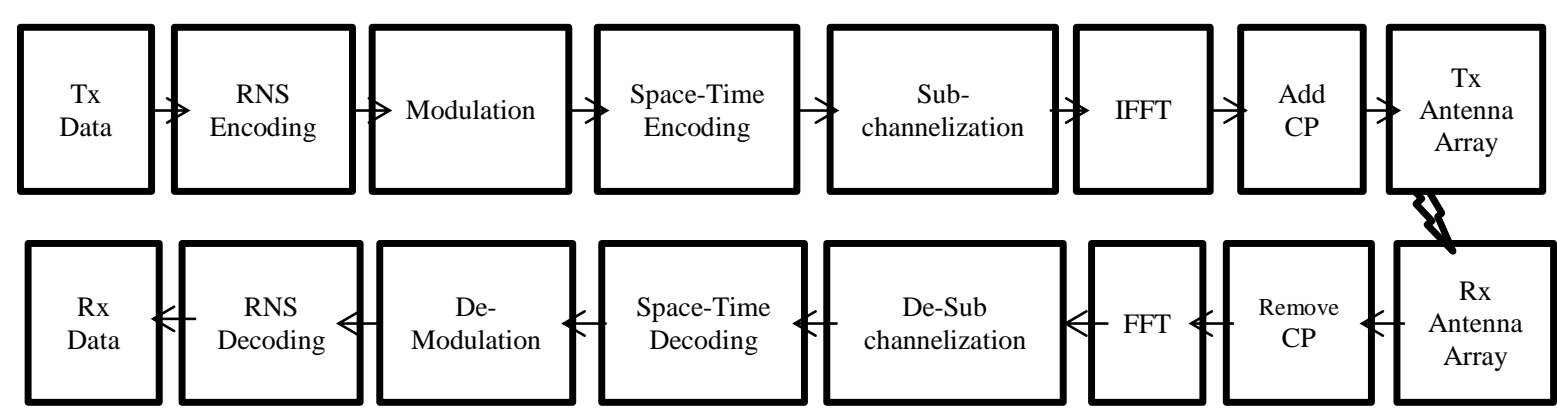

Figure 2. MIMO-RNS-OFDM system model

Before transmission, a Cyclic prefix (CP) is added to decrease the effect of Inter Symbol Interference (ISI) and Inter Carrier Interference (ICI) caused by the multipath channel [14, 15]. This CP is a copy of the last section of the OFDM symbol that is attached to the front of transmitted OFDM symbol. To represent the satellite channel in the model presented in Figure 2, several channel fading are given; starting with Additive White Gaussian Noise (AWGN) channel, and then adding multipath fading factors through using Rician, Rayleigh and Rice-Lognormal distribution (RLN) distribution fading models [16]. The receiver blocks are the reverse blocks of the transmitter.

\section{IMAGE QUALITY MEASURES}

In this paper, an image is transmitted over the communication channel using the RNS-based transceiver system provided in section 5. Consequently, this section provides the key parameters that are utilized to measure and evaluate the quality of the received image as a way of analyzing the system performance. These parameters are the Peak Signal to Noise Ratio (PSNR), Mean Square Error (MSE) and the Correlation coefficient (CORR) as seen in the following subsections.

\subsection{Mean squared error}

The MSE [17] is a measure of the squares of the difference between the intensities of the received and the original image, and is represented as seen in (9):

$$
\mathrm{MSE}=\frac{1}{\mathrm{MN}} \sum_{\mathrm{i}=1}^{\mathrm{M}} \sum_{\mathrm{J}=1}^{\mathrm{N}}\left(\mathrm{S}_{\mathrm{i}, \mathrm{J}}-\mathrm{C}_{\mathrm{i}, \mathrm{J}}\right)^{2}
$$

where;

$\mathrm{M} \& \mathrm{~N}$ denote total number of the pixels in the horizontal / vertical dimensions of the image respectively. $\mathrm{C}_{\mathrm{i}, \mathrm{J}} \& \mathrm{~S}_{\mathrm{i}, \mathrm{J}}$ represent pixel of the original and received image respectively. 


\subsection{The peak signal to noise ratio (PSNR)}

The PSNR as seen in (10) is the difference between the received and the original image, taking the original image as a reference [18].

$$
\text { PSNR }=10 \log _{10}\left(\frac{\mathrm{I}_{\max }^{2}}{\mathrm{MSE}}\right) \mathrm{dB}
$$

where $I_{\max }$ is equal to 255 for 8 bit sampling images.

The PSNR is an approximation to human perception of reconstruction quality, where a typical value of lossless image is $30-50 \mathrm{~dB}$ and acceptable values in wireless communication are between 20 to $25 \mathrm{~dB}$. In the absence of noise, the original and received images are identical, giving a MSE equal to zero, and in this case the PSNR is infinite.

\subsection{The correlation coefficient (CORR)}

The cross-correlation coefficient (CORR) is given by (11) as follows:

$$
\text { Corr }=\frac{\sum_{\mathrm{i}=1 \mathrm{~J}=1}^{\mathrm{M}} \sum_{\mathrm{i}, \mathrm{J}}^{N}\left(\mathrm{C}_{\mathrm{C}}\right)\left(\mathrm{S}_{\mathrm{i}, \mathrm{J}}-\overline{\mathrm{S}}\right)}{\sqrt{\sum_{\mathrm{i}=1 \mathrm{~J}=1}^{\mathrm{M}} \sum_{\mathrm{J}=\mathrm{J}}^{N}\left(\mathrm{C}_{\mathrm{J}}-\overline{\mathrm{C}}\right) \sum_{\mathrm{i}=1 \mathrm{~J}=1}^{\mathrm{M}} \sum_{\mathrm{J}, \mathrm{J}}^{N}\left(\mathrm{~S}_{\mathrm{S}}-\overline{\mathrm{S}}^{2}\right.}}
$$

where $\overline{\mathrm{C}} \& \overline{\mathrm{S}}$ Represent the pixel of the original and recieved image respectively.

\section{SIMULATION RESULTS}

Performance of the proposed system was investigated using MATLAB tool, and is performed through transmitting an image with a FEC coding scheme, modulated and multiplexed through OFDM system and add to it a 1/8 CP, over different fading channels. The use of RNS as a coding scheme in the transmission system is evaluated with respect to non-RNS system, over various fading channels and different modulation index. Moreover, the RRNS is simulated and its performance as an FEC scheme is evaluated with respect to LDPC coders that are currently used in wireless communication systems.

\subsection{Transmitted and received images in non-OFDM system}

In a non-OFDM wireless system, where several images shown in Figure 3 are transmitted using 512QAM modulation scheme, with low SNR $(5 \mathrm{~dB})$, and recording the MSE, PSNR and CORR for the received image, over AWGN, Rician, and Rayleigh fading models as shown in Tables 2-4.
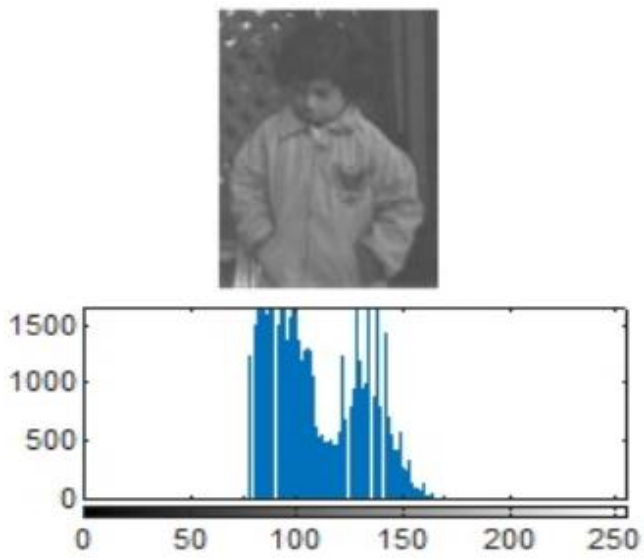
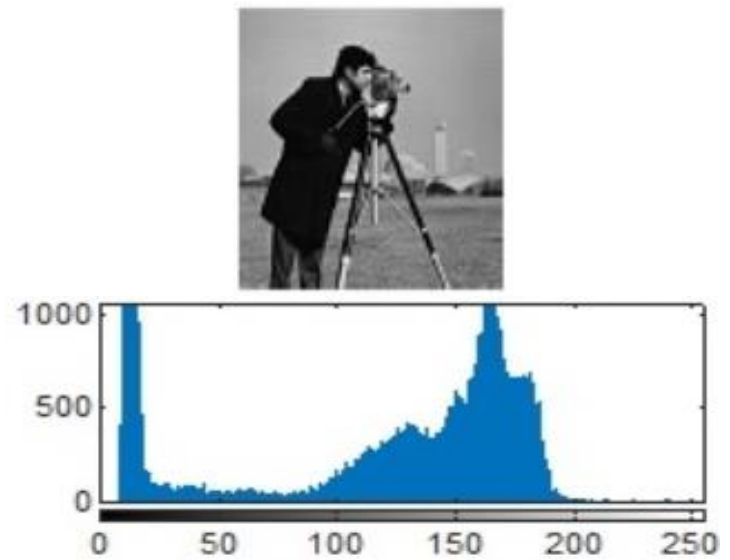

Figure 3. Transmitted 'pout'and 'cameraman' images and their associated histograms 
Table 2. System MSE for 1024-QAM communication system

\begin{tabular}{ccccc}
\hline \multicolumn{2}{c}{ Communication System } & \multicolumn{3}{c}{ Mean Square Error Function } \\
& & AWGN model & Rician model & Rayleigh model \\
\hline 'pout' & Non-RNS & 803 & 810 & 841 \\
size 240*291 & RNS & 28.4 & 23 & 25 \\
'cameraman' & Non-RNS & 721 & 741 & 738 \\
size 256*256 & RNS & 21.9 & 26 & 22.2808 \\
\hline
\end{tabular}

Table 3. System PSNR for 1024-QAM communication system

\begin{tabular}{|c|c|c|c|c|}
\hline \multicolumn{2}{|c|}{ Communication System } & \multicolumn{3}{|c|}{ PSNR in $\mathrm{dB}$} \\
\hline & & AWGN model & Rician model & Rayleigh model \\
\hline 'pout' & Non-RNS & 19.08 & 19.02 & 18.879335 \\
\hline size $240 * 291$ & RNS & 33.5969 & 34.576 & 34.213 \\
\hline 'cameraman' & Non-RNS & 19.5467109 & 19.43112 & 19.447675 \\
\hline size $256 * 256$ & RNS & 34.7159 & 33.9795 & 34.651476 \\
\hline
\end{tabular}

Table 4. The correlation between received and transmitted image

\begin{tabular}{ccccc}
\hline \multicolumn{2}{c}{ Communication System } & & Correlation Factor \\
& & AWGN model & Rician model & Rayleigh model \\
\hline 'pout' & Non-RNS & 0.6351853 & 0.633 & 0.6386091 \\
size $240 * 291$ & RNS & 0.974492 & 0.979 & 0.977704 \\
'cameraman' & Non-RNS & 0.9083817 & 0.905862 & 0.906193 \\
size 256*256 & RNS & 0.9971786 & 0.996658 & 0.9971371 \\
\hline
\end{tabular}

From the above Tables 2-4, it has shown the enhancement done by the usage of the RNS code in a non-OFDM communication system, which is attributed to the selection of the RNS moduli's to represent the same information using less data symbols, leading to increase in the energy per symbol and consequently the reduction of the system BER.

\subsection{BER and PAPR performance for RNS as a coding scheme}

In a MIMO-OFDM system, the system BER and PAPR are used to measure and analyze the system performance as seen in Figure 4 and Figure 5. From Figure 4, it is shown an enhancement of around 4-5 dB in the SNR at a BER $=10^{-3}$ compared to non-RNS coding scheme. Where the RNS coding gain could be explained when the bit-wide coefficient $=\log _{2} \mathrm{M}_{\mathrm{i}} / \log _{2 \mathrm{Mary}}>1$, the total number of bits per residue symbol is also bigger than the number of bits of the M-ary input, implying that the energy per symbol increases. Hence, the bandwidth is decreased or for the same bandwidth the BER decreases. In Figure 5, it is seen from 5-6 dB reduction in the PAPR achieved through converting the input data to RNS representation. This is attributed to the fact that the residue is always smaller than the corresponding modulus, which may be chosen smaller than the original number, thus the residue is smaller than the original number. Hence, the PAPR for RNS scheme is lower than that without RNS coding.
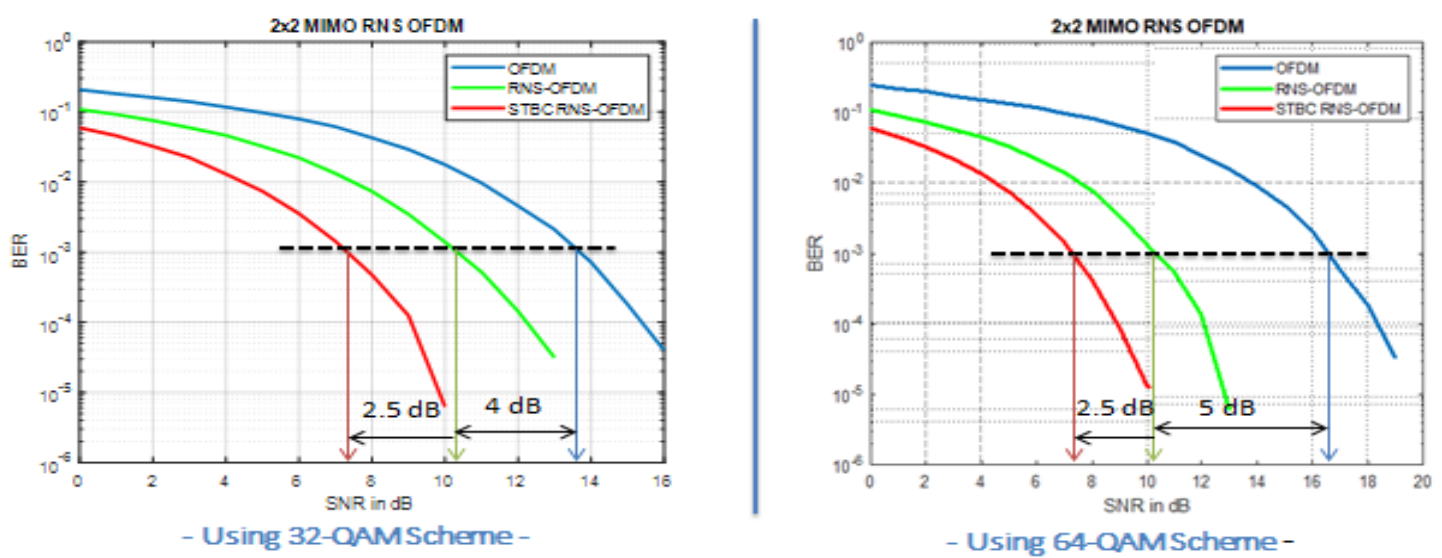

Figure 4 (a). $2 * 2$ MIMO-OFDM BER over AWGN channel 

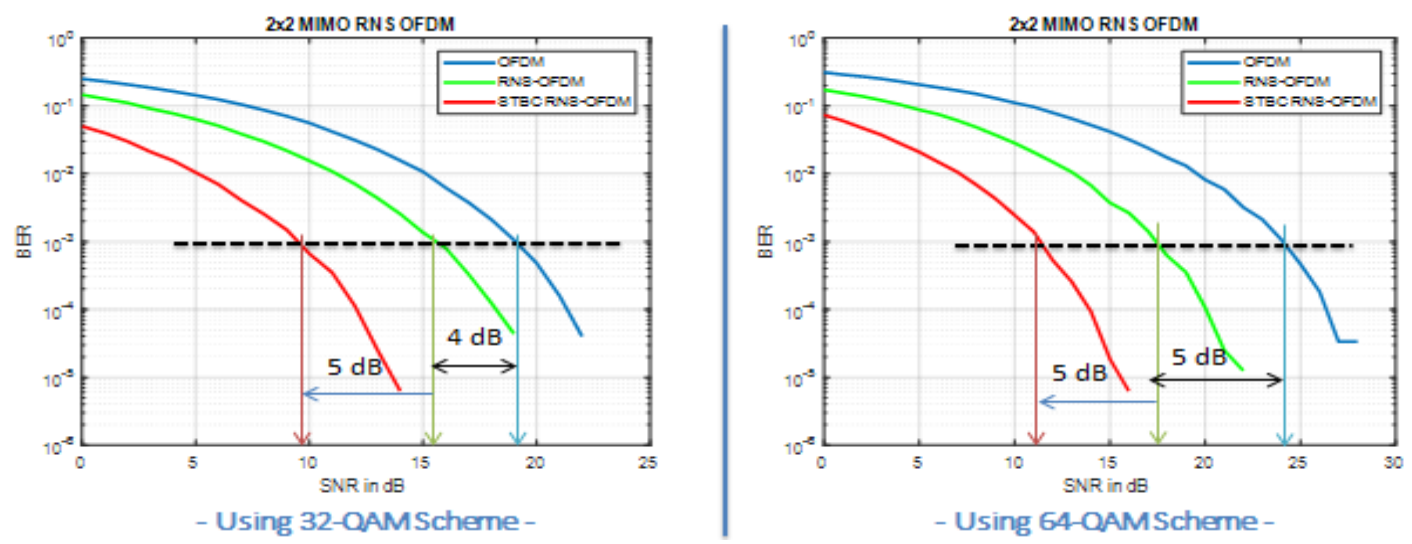

Figure 4 (b). $2 * 2$ MIMO-OFDM BER over Rayleigh channel

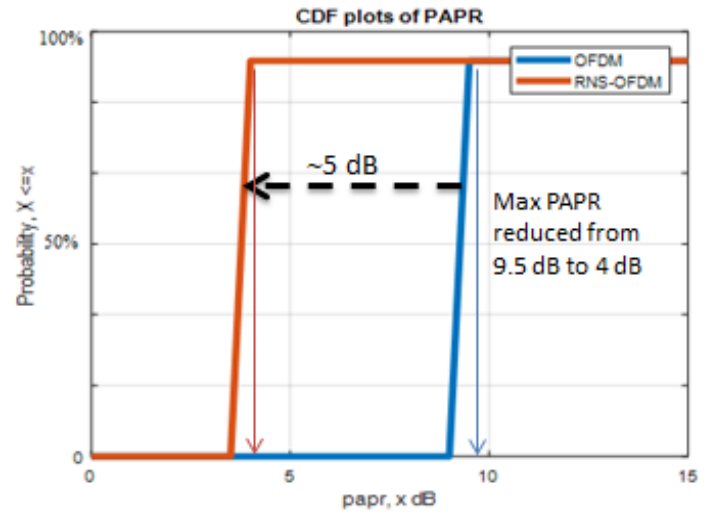

- Number of Transmitted symbols $=5000$ -

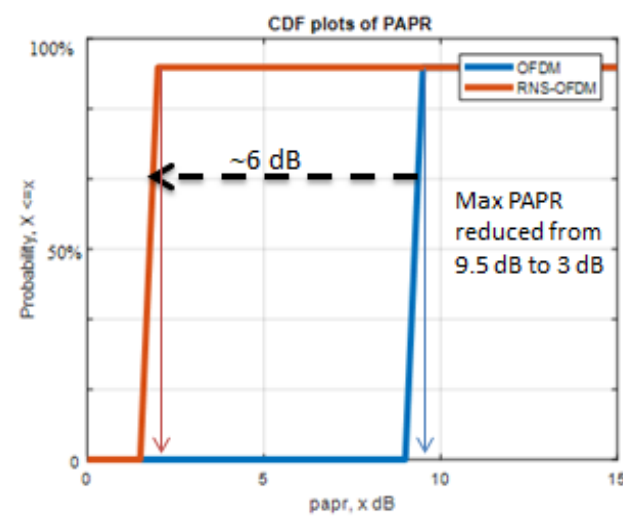

- Number of Transmitted symbols $=10,000-$

Figure 5. 2*2 MIMO-OFDM PAPR evolutions

\subsection{The transmitted and received images in MIMO-OFDM system}

Transmit different images using 1024-QAM and coding it with RNS moduli $\left\{\begin{array}{llll}11 & 9 & 7 & 5\end{array}\right\}$, over different fading channel as: AWGN, Rician, and Rayleigh models, and recording the received image and its associated histogram as seen in Figure 6. From Figure 6, it is shown that over different fading channels, the received image and its associated histogram is enhanced using RNS coding compared to non-RNS coding scheme.

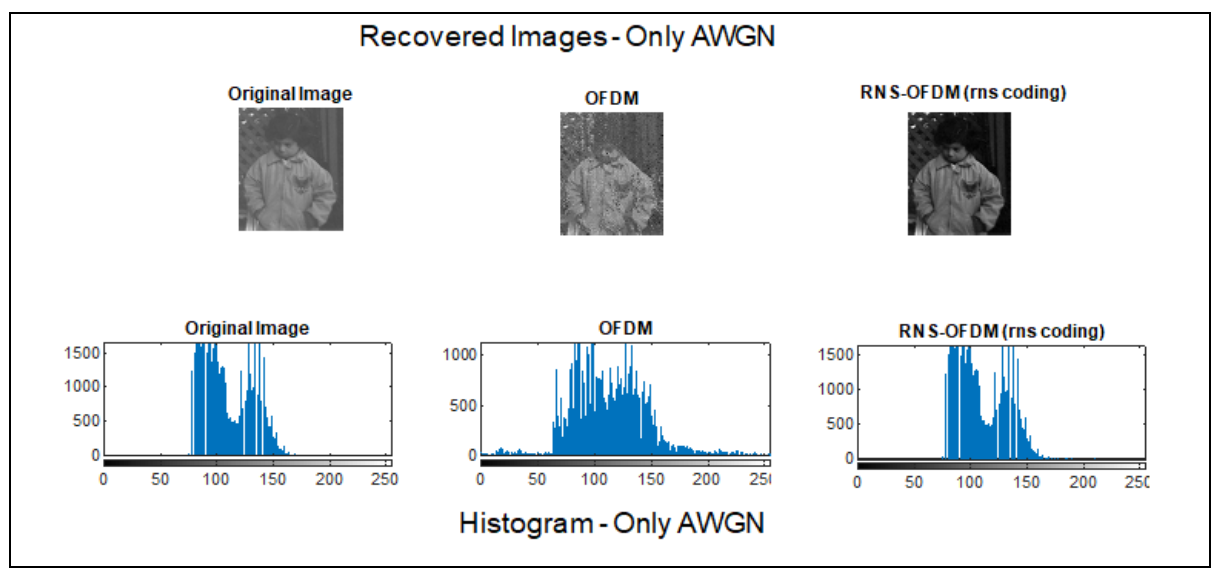

Figure 6(a). Original and recovered image and its histogram over AWGN channel 


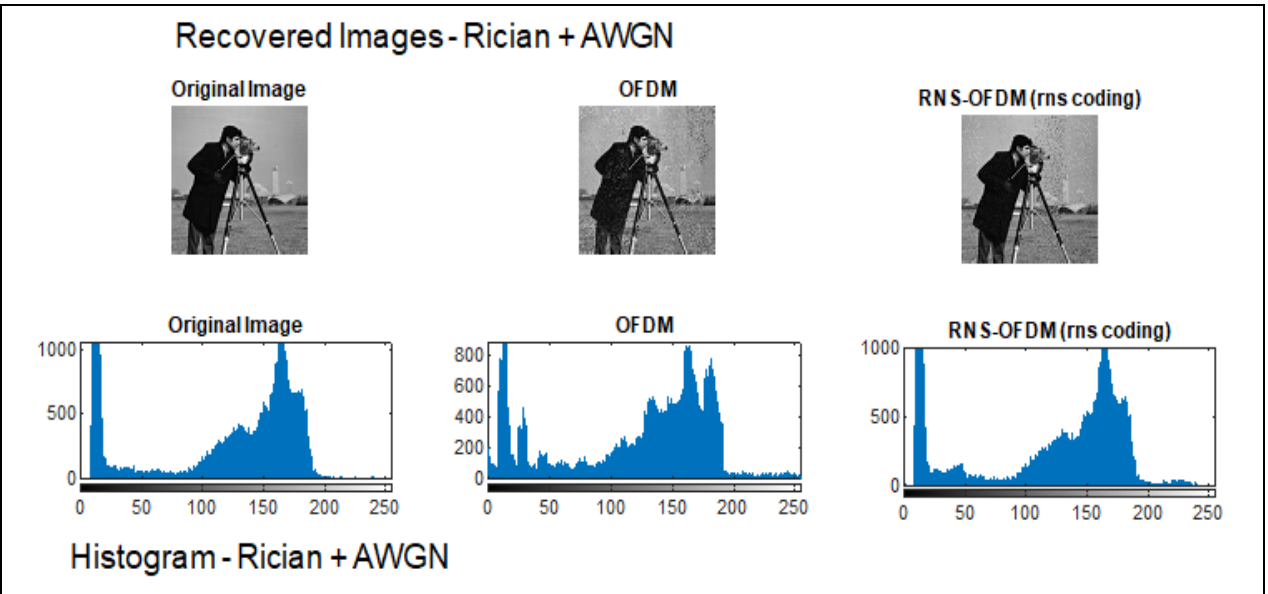

Figure 6(b). Original and recovered image and its histogram over rician channel

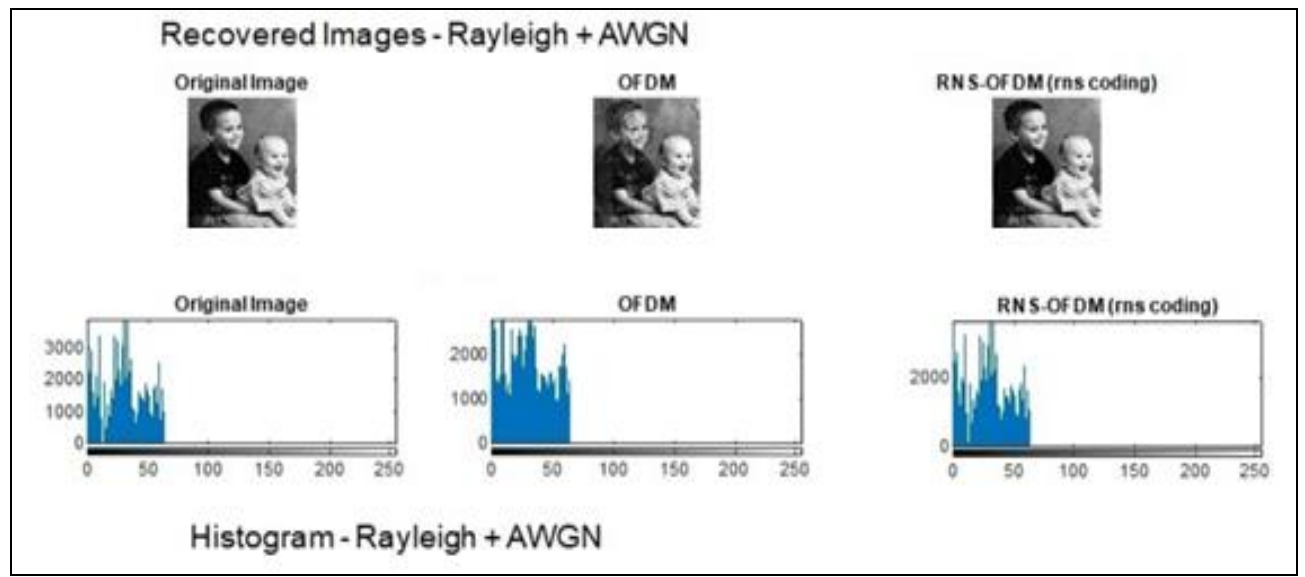

Figure 6(c). Original and recovered image and its histogram over Rayleigh channel

\subsection{The received MSE and PSNR in MIMO-OFDM system over various fading models}

Transmit the same images seen previously in Figure 3 in a MIMO-OFDM communication system with SNR equal to $20 \mathrm{~dB}$, using 1024-QAM modulation scheme, and recording the MSE, and PSNR for the received image over different fading channel models as: LOS, AWGN, Rician, and Rayleigh models, as shown in Table 5 and 6.

Table 5. System MSE for 1024-QAM communication system

\begin{tabular}{cccccc}
\hline \multicolumn{2}{c}{ Communication System } & \multicolumn{4}{c}{ Mean Square Error Function } \\
\hline 'pout' & OFDM & 681 & 647 & 761 & Rayleigh model \\
\hline size $240 * 291$ & RNS-OFDM & 2.9 & 1.17 & 103 & 110 \\
'cameraman' & OFDM & 771 & 593 & 588 & 833 \\
size $256 * 256$ & RNS-OFDM & 79 & 0 & 7.24 & 58.8 \\
\hline
\end{tabular}

Table 6. System PSNR for 1024-QAM communication system

\begin{tabular}{cccccc}
\hline \multicolumn{2}{c}{ Communication System } & \multicolumn{4}{c}{ PSNR in dB } \\
& & LOS model & AWGN model & Rician model & Rayleigh model \\
\hline 'pout' & OFDM & 19.79 & 20.02 & 19.316 & 19.04595342 \\
size 240*291 & RNS-OFDM & 43.4 & 47.43 & 27.98 & 27.71687676 \\
'cameraman' & OFDM & 19.25 & 20.39 & 20.433 & 18.922 \\
size 256*256 & RNS-OFDM & 29 & 99 & 39.527 & 30.43 \\
\hline
\end{tabular}


As seen in Table 5, the MSE is reduced over various fading schemes when the RNS coding is implemented, which indicate the advantage of RNS coding in reducing the transmission errors, and in Table 6, the PSNR is increased indicating the increasing coherency of received image with respect to the original one when using RNS coding scheme.

Consequently, from the Table 5 and 6, it shown the enhancement encountered when using RNS as a coding scheme, which is attributed to both the reduction in PAPR as seen in section 7.2 and the ability to reduce the amount of transmitted information without losing any data through the RNS coding scheme as seen in section 7.1 .

\subsection{The cross-correlation for OFDM and RNS-OFDM systems}

Another key parameter used to estimate the received image quality is through measuring the crosscorrelation factor between the received and transmitted image. In Table 7, a record for the correlation of various images as 'cameraman', 'pout' and 'kids' transmitted using 1024-QAM has been provided. From Table 7, shown that the use of RNS coding would increase the correlation factor more close to ' 1 ', which is an indication of the enhanced received image in comparison to non-RNS coding system. In the coming sections 7.6 to 7.8 the RRNS FEC scheme is simulated, analyzed and compared to conventional FEC techniques through recording and evaluating the BER, PAPR, MSE, PSNR, and CORR parameters.

Table 7. The correlation between received and transmitted image

\begin{tabular}{cccccc}
\hline \multicolumn{2}{c}{ Communication System } & \multicolumn{4}{c}{ Correlation Factor } \\
& & LOS model & AWGN model & Rician model & Rayleigh model \\
\hline 'pout' & OFDM & 0.665724729 & 0.67539320593 & 0.64099311611 & 0.639 \\
size 240*291 & RNS-OFDM & 0.997300542 & 0.99890938 & 0.914315425689 & 0.99285 \\
'cameraman' & OFDM & 0.9019447 & 0.92440049682 & 0.92570339 & 0.8939285 \\
size 256*256 & RNS-OFDM & 0.98986 & 1 & 0.99906752 & 0.99246663 \\
\hline
\end{tabular}

\subsection{BER performance for RRNS as FEC scheme in MIMO-OFDM system}

The BER performance is evaluated using $\operatorname{RNS}$ coding $\{3,5,7\}$ with redundant moduli $\{11\}$, for MIMO-OFDM system as an error correction method compared to conventional DVB-S2 FEC technique that use LDPC scheme and non FEC schemes. From the Figure 7, it shown that error correction scheme with redundant RNS provide a comparable performance with that using LDPC scheme that is currently used in DVB-S2 systems, and about $6 \mathrm{~dB}$ SNR reduction at a BER $=10^{-3}$ compared to non-FEC scheme.
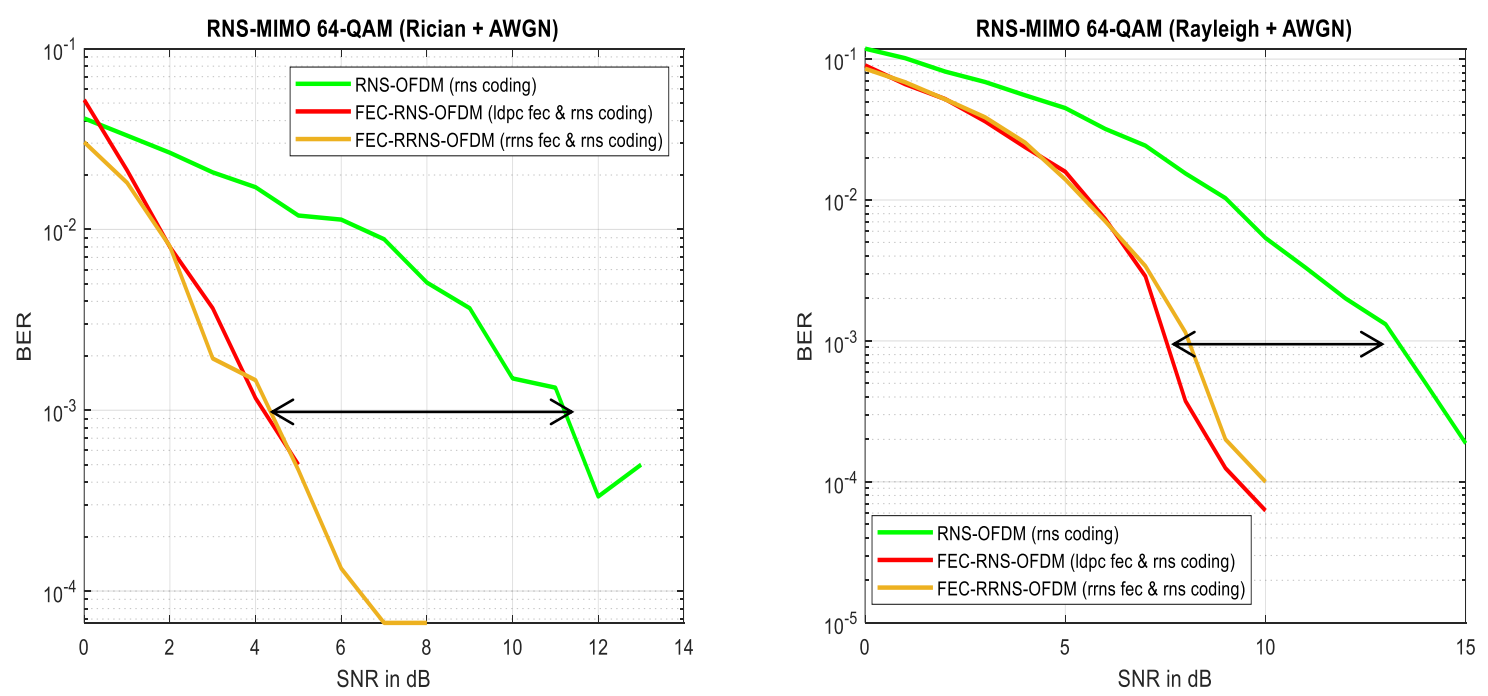

Figure 7. MIMO-OFDM system performance with FEC Schemes 


\subsection{PAPR performance for MIMO-OFDM System with RRNS as FEC}

For a MIMO-OFDM system over Rayleigh fading model channel, the PAPR for the transmitted signal is as shown in Figure 8. From the Figure 8, it shown that the system with RRNS has the minimum signal amplitude by about $20 \%$ compared to LDPC FEC scheme (FEC-RNS-OFDM).

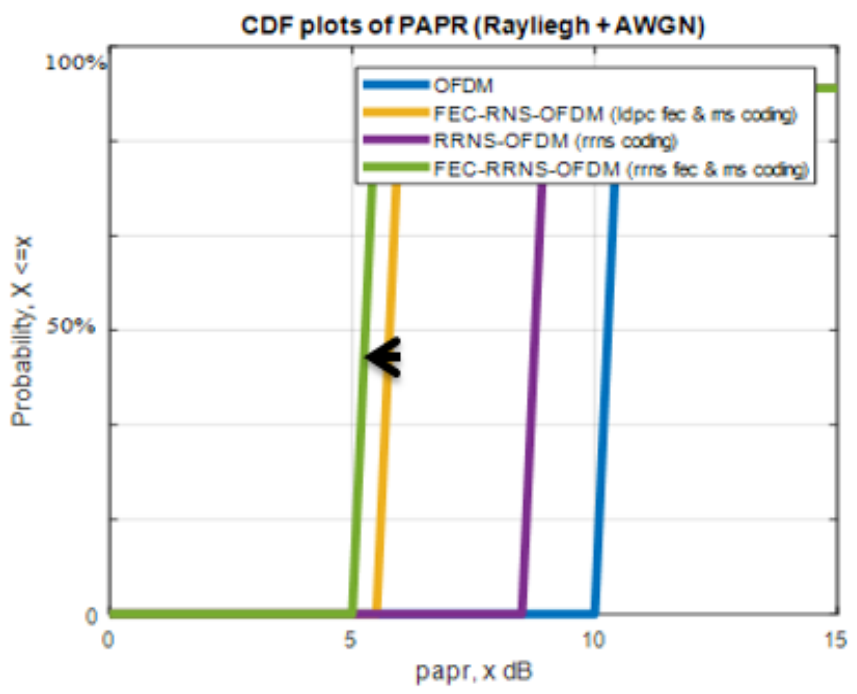

Figure 8. PAPR measurement for MIMO-OFDM system

\subsection{The RRNS performance using image quality measurements}

Transmitting an image coded using RRNS that have an information moduli's $\left\{\begin{array}{llll}11 & 9 & 7 & 5\end{array}\right\}$ and redundant moduli $\{13\}$, and modulated using 1024-QAM, through a Rician and Rayleigh fading channels, and measuring the MSE, PSNR and correlation factor and comparing it with the same communication system without FEC scheme, as shown in Table 8 and 9. From Table 8 and 9, it shown the reduction of generated errors, increased SNR and correlation factor when RNS is used as a FEC scheme, compared to RNS as a coding technique only and without using RNS in the communication system.

Table 8. System MSE/PSNR/correlation over rician channel

\begin{tabular}{ccccc}
\hline \multicolumn{2}{c}{ Communication System } & \multicolumn{3}{c}{ Received Image Quality Indicators } \\
& OFDM & 761 & 19.316 & Correlation Factor \\
\hline \multirow{2}{*}{ 'pout' } & RNS-OFDM & 103 & 27.98 & 0.64099311611 \\
size 240*291 & FEC-RNS-OFDM & 0 & 99 & 0.914315425689 \\
& & & & 1 \\
\hline
\end{tabular}

Table 9. System MSE/PSNR/correlation over Rayleigh channel

\begin{tabular}{ccccc}
\hline \multirow{2}{*}{ Communication System } & \multicolumn{3}{c}{ Received Image Quality Indicators } \\
& & MSE & PSNR & Correlation Factor \\
\hline \multirow{2}{*}{ 'pout' } & OFDM & 889 & 18.64 & 0.63052 \\
size 240*291 & RNS-OFDM & 339 & 22.8 & 0.769714 \\
& FEC-RNS-OFDM & 0.51310137 & 51 & 0.99952286 \\
\hline
\end{tabular}

\section{CONCLUSION}

The utilization of RNS coding system with parallel distributed arithmetic and with no dependence between the different arithmetic blocks would simplify the overall design and reduces the complexity of the individual building blocks. In this paper, RNS as a coding scheme is used to exploit the quality of image transmission through OFDM technique that encodes digital data over multiple carrier frequencies. Through this coding algorithm, it was able to reduce the transmitted signal dynamic range such that the obtained PAPR was around $20 \%$ less compared to the conventional MIMO-OFDM system, as well as enhancing the overall SNR of the system. 
Using RNS coding scheme, a reduction of 2-5 dB SNR @ BER $=10^{-3}$ depending on the modulation index is achieved. This indicates the RNS gain over non-RNS systems, through the measure of Signal-toNoise Ratio (SNR), which attributed to the "coding" gain of RNS that decrease the cross correlation between transmitted symbols, reducing interference and thus enhance system performance. The paper provides the enhancement done on transmitting an image in a communication system using RNS as both coding and error detection and correction scheme. Where, the system MSE had been reduced, the PSNR increased significantly and the correlation factor nearly reached to unity.

\section{REFERENCES}

[1] H. Bolcskei, "MIMO-OFDM wireless systems: basics, perspectives, and challenges," IEEE Wireless Communications, vol. 13, pp. 31-37, 2006.

[2] R. Biradar, "Study and Analysis of 2x2 MIMO Systems for Different Modulation Techniques Using MATLAB," techrepublic, 2015, [Online], Available: http://www.techrepublic.com/resource-library/whitepapers/study-andanalysis-of-2x2-mimo-systems-for-different-modulation-techniques-using-matlab/.

[3] Ghassan M. T. A., "Orthogonal Frequency Division Multiplexing. Theory and Challenges," UofKEJ, vol. 1, pp. 1-8, 2011.

[4] R. F. Fischer and M. Hoch, "Peak-to-average power ratio reduction in mimo ofdm," Communications, 2007. ICC'07. IEEE International Conference on. IEEE, 2007, pp. 762-767.

[5] L. Wang, "Cooperative pts for papr reduction in mimo-ofdm," Electronics Letters, vol. 47, pp. 351-352, 2011.

[6] M. Roshanzade, "Using Residue Number Systems for Improving QoS and Error Detection \& Correction in Wireless Sensor Networks," ICCSN, 2011 IEEE 3rd International Conference on, 2011, pp. 1-5.

[7] P. Maji, "Application of Residue Arithmetic in Communication and Signal Processing," Master of Science, National Institute of Technology, India, 2011.

[8] A. James, et al., "Multiple error correction using non-binary Redundant Residue Number System," India Conference (INDICON) 2015, 2015.

[9] H. Krisna, "A coding Theory Approach to Error Control in Redundant Residue Number Systems," IEEE Transactions on Circuits and Systems II: Analog and Digital Signal Processing, vol. 39, pp. 8-17, 1992.

[10] S. A. Mumin, "An Improved Redundant Residue Number System Based Error Detection and Correction Scheme for the Moduli Set," Advances in Wireless Communications and Networks journal, 2016.

[11] J. Singh and J. Singh, "A Comparative study of Error Detection And Correction Coding Techniques," 2012 Second International Conference on Advanced Computing \& Communication Technologies, 2012.

[12] DVB-S2. EN 302307 V1.1.2 (06/06), "Digital Video Broadcasting; Second generation framing structure, channel coding and modulation systems for Broadcasting, Interactive Services, News Gathering and other broadband satellite applications," ETS, 2006.

[13] T. O. Olatunde and A. G. Kazeem, "Redundant Residue Number System Based Fault Tolerant Architecture over Wireless Network," CoRI'16, Ibadan, Nigeria, 2016.

[14] S. Kaur, "Orthogonal Frequency Division Multiplexing in Wireless Communication Systems: A Review," International Journal of Advanced Research in Computer Engineering \& Technology, vol. 1, 2012.

[15] D. C. Shah, et al., "Effects of cyclic prefix on OFDM system," Proceedings of the ICWET '10 International Conference \& Workshop on Emerging Trends in Technology, Mumbai, Maharashtra, India, 2010, pp. 420-424.

[16] F. Vatalaro and F. Mazzenga, "The generalized Rice lognormal channel model—first and second order statistical characterization and simulation," International Journal of Satellite Communications, pp. 29-45, 2002.

[17] W. Bender, et al., "Techniques for data hiding," IBM Syst. J., vol. 35, pp. 313-336, 1996.

[18] K. Silpa and S. A. Mastani, "Comparison of Image Quality Metrics," The International Journal of Engineering Research and Technology (IJERT), vol. 1, 2012. 